\title{
MAGNETOHYDRODYNAMIC EFFECTS IN PROPAGATING RELATIVISTIC JETS: REVERSE SHOCK AND MAGNETIC ACCELERATION
}

\author{
Yosuke Mizuno $^{1,2}$, Bing Zhang ${ }^{2}$, Bruno Giacomazzo ${ }^{3}$, Ken-Ichi Nishikawa ${ }^{1}$, Philip E. Hardee ${ }^{4}$, Shigehiro Nagataki ${ }^{5}$, \\ AND DiETER H. HARTMANN ${ }^{6}$ \\ ${ }^{1}$ Center for Space Plasma and Aeronomic Research, University of Alabama in Huntsville, NSSTC, 320 Sparkman Drive, Huntsville, AL 35805, USA; \\ mizuno@cspar.uah.edu \\ 2 Department of Physics and Astronomy, University of Nevada, Las Vegas, NV 89154, USA \\ ${ }^{3}$ Max-Plank-Institut für Gravitationsphysik, Albert-Einstein-Institut, Potsdam-Golm, Germany \\ ${ }^{4}$ Department of Physics and Astronomy, University of Alabama, Tuscaloosa, AL 35487, USA \\ ${ }^{5}$ Yukawa Institute for Theoretical Physics, Kyoto University, Sakyo, Kyoto, Japan \\ ${ }^{6}$ Department of Physics and Astronomy, Clemson University, Clemson, SC 29634, USA \\ Received 2008 August 15; accepted 2008 October 31; published 2008 December 8
}

\begin{abstract}
We solve the Riemann problem for the deceleration of an arbitrarily magnetized relativistic flow injected into a static unmagnetized medium in one dimension. We find that for the same initial Lorentz factor, the reverse shock becomes progressively weaker with increasing magnetization $\sigma$ (the Poynting-to-kinetic energy flux ratio), and the shock becomes a rarefaction wave when $\sigma$ exceeds a critical value, $\sigma_{c}$, defined by the balance between the magnetic pressure in the flow and the thermal pressure in the forward shock. In the rarefaction wave regime, we find that the rarefied region is accelerated to a Lorentz factor that is significantly larger than the initial value. This acceleration mechanism is due to the strong magnetic pressure in the flow. We discuss the implications of these results for models of gamma-ray bursts and active galactic nuclei.
\end{abstract}

Key words: galaxies: jets - gamma rays: bursts - MHD - shock waves - relativity

\section{INTRODUCTION}

Relativistic jets are believed to exist in active galactic nuclei (AGNs), black hole binaries, and gamma-ray bursts (GRBs), but their composition is still poorly understood. It has been argued that magnetic fields could play an important dynamic role in these jets (e.g., Lovelace 1976; Blandford 1976; Blandford \& Znajek 1977; Blandford \& Payne 1982; Usov 1992; Thompson 1994; Mészáros \& Rees 1997; Lyutikov \& Blandford 2003; Vlahakis \& Königl 2003, 2004), but the degree of magnetization, quantified by the magnetization parameter $\sigma$ (the ratio of electromagnetic to kinetic energy flux), is poorly constrained by observations. GRB afterglow modeling indicates that the ejecta are more magnetized than the ambient medium, suggesting a possibly important dynamic role for magnetic fields in GRB jets (Fan et al. 2002; Zhang et al. 2003; Kumar \& Panaitescu 2003; Gomboc et al. 2008).

A useful diagnostic for the degree of jet magnetization can be obtained from the interaction between the decelerating jet and the ambient medium. Added magnetic field pressure in the jet alters the condition for the formation of a reverse shock (RS) as well as its strength (Kennel \& Coroniti 1984). Analytical studies of the deceleration of a GRB fireball with arbitrary magnetization (Zhang \& Kobayashi 2005, hereafter ZK05; see also Fan et al. 2004 for $\sigma \leqslant 1$ ) suggest novel behavior that does not exist in pure hydrodynamic (HD, $\sigma=0$ ) models (Sari \& Piran 1995; Kobayashi et al. 1999). However, consensus on the conditions required for the existence of the RS or how Poynting flux is transferred to kinetic flux in the interaction region has not yet been achieved (ZK05; Lyutikov 2006; Giannios et al. 2008). We present a one-dimensional study of the interaction between a magnetized relativistic flow and a static, unmagnetized external medium. A Riemann problem is solved both analytically and numerically over a broad range of $\sigma$.

\section{THE RIEMANN PROBLEM}

We consider a Riemann problem consisting of two uniform initial states (left and right) with discontinuous HD properties specified by the rest-mass density $\rho$, gas pressure $p$, specific internal energy $u$, specific enthalpy $h \equiv 1+u / \rho c^{2}+p / \rho c^{2}$, and normal velocity $v^{N}$. The right state (the medium external to the jet) is assumed to be a cold fluid with constant density, at rest. Specifically, we select the initial conditions: $\rho_{R}=1.0 \rho_{0}$, $p_{R}=10^{-2} \rho_{0} c^{2}, v_{R}^{N}=v_{R}^{x}=0.0$, where $\rho_{0}$ is an arbitrary normalization constant (our simulations are scale-free) and $c$ is the speed of light. The left state (the propagating relativistic flow) is assumed to have a higher density and pressure than the right state, as well as a relativistic velocity. Specifically, $\rho_{L}=10^{2} \rho_{0}, p_{L}=1.0 \rho_{0} c^{2}$, and $v_{L}^{N}=v_{L}^{x}=0.995 c\left(\gamma_{L} \simeq 10\right)$. The fluid is described by an adiabatic equation of state $p \propto \rho^{\Gamma}$ with $\Gamma=4 / 3$.

To investigate the effects of magnetic fields, we consider a perpendicular field component in the jet with $B^{y}=31.623$, $100.0,316.23$, and 447.21 in units of $\left(4 \pi \rho_{0} c^{2}\right)^{1 / 2}$ measured in the laboratory frame, corresponding to $\sigma \equiv B^{2} / 4 \pi \gamma^{2} h \rho c^{2} \simeq$ $B^{2} / 4 \pi \gamma^{2} \rho c^{2}$ being $0.1,1.0,10.0$, and 20.0 , respectively. This field is motivated by the predicted toroidal field domination at the deceleration radius for GRB outflows (e.g., Spruit et al. 2001; ZK05). Increasing $\sigma$ increases the total (kinetic plus magnetic) energy density of the left (jet) state.

\section{RESULTS}

We calculate exact solutions of this problem, using the code of Giacomazzo \& Rezzolla (2006), in the region $0.8 \leqslant x \leqslant 1.2$ with an initial discontinuity at $x=1.0$, where $x$ is in arbitrary units. 
(a)

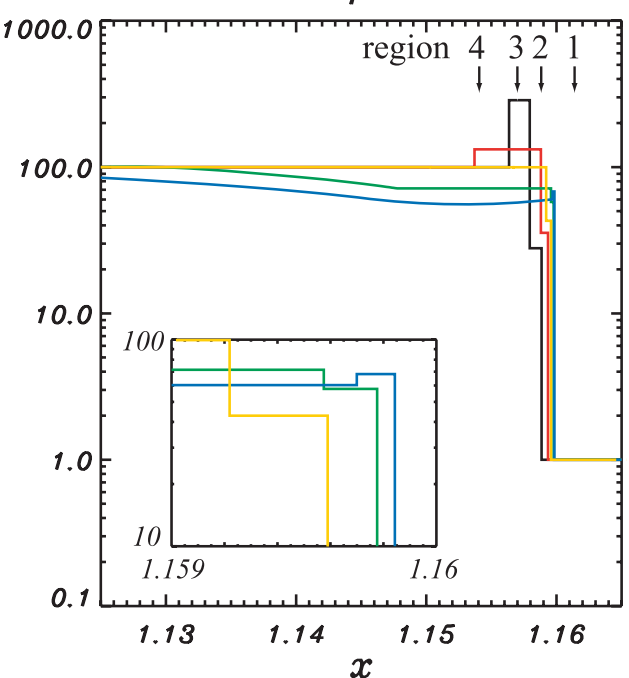

(c)

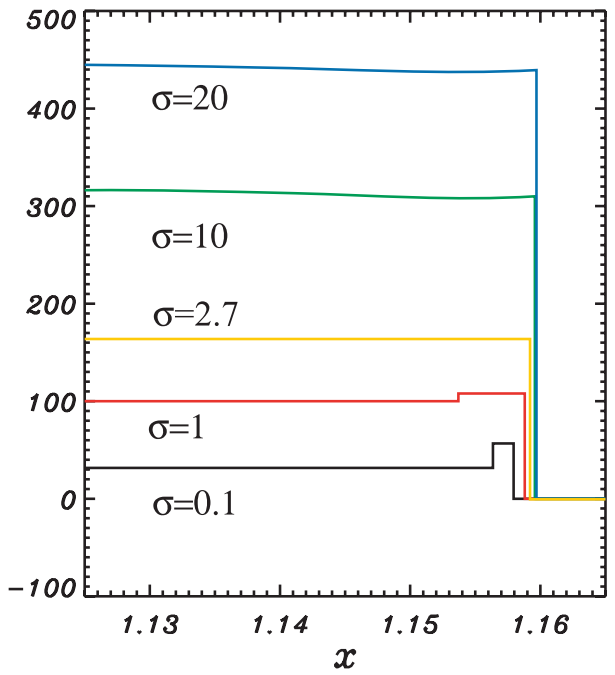

(b)

$p$

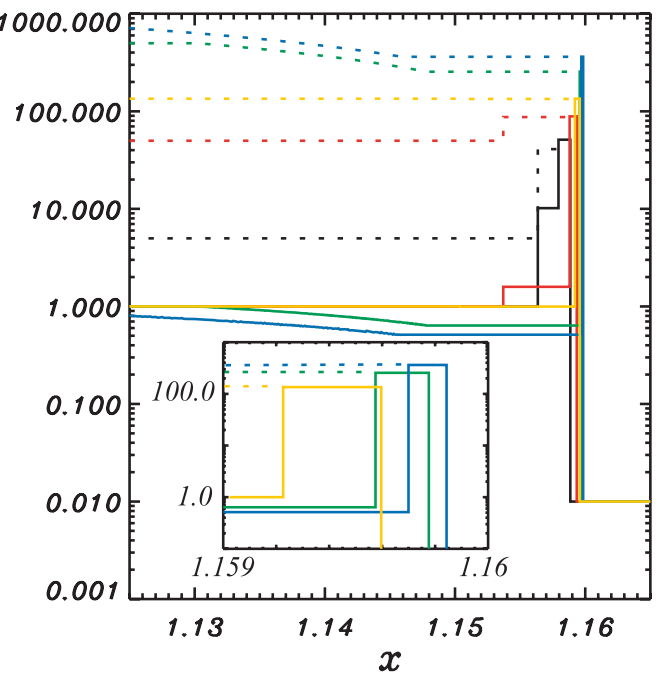

(d)

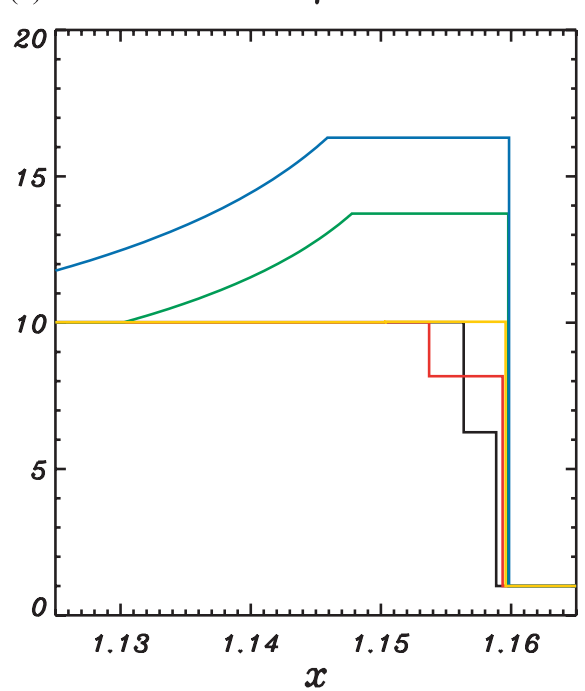

Figure 1. Profiles of (a) density, (b) gas pressure (solid lines) and magnetic pressure (dotted lines), (c) magnetic field $\left(B^{y}\right)$, and (d) Lorentz factor $(\gamma)$ of $\sigma=0.1$ (black), 1.0 (red), 10.0 (green), and 20.0 (blue) cases at time $t=0.16$. Other parameters: $\rho_{L}=100.0, \rho_{R}=1.0, \gamma_{L}=10.0$. The critical value, $\sigma_{c} \simeq 2.7$, case is shown as yellow lines. Close-up forward shock regions are inserted. Arrows indicate four physically distinct regions: (1) unshocked medium, (2) shocked medium, (3) shocked flow, and (4) unshocked flow corresponding to the $\sigma=0.1$ case.

\subsection{Flow-Medium Interaction}

The exact solutions are presented in Figure 1. The four panels display profiles of the gas density, gas (and magnetic) pressure, magnetic field strength $B^{y}$, and the Lorentz factor at time $t=0.16$. $^{7}$ Different colors represent different $\sigma$ values: 0.1 (black), 1.0 (red), 2.7 (yellow), 10.0 (green), and 20.0 (blue). The initial Lorentz factor of the left state (jet) is $\gamma_{L}=10$.

For $\sigma=0.1$ (black), the solution shows a right-moving fast shock (FS: forward shock; $S_{\rightarrow}$ ) and a left-moving fast shock (RS: reverse shock; $\leftarrow S$ ) relative to the contact discontinuity $(C)$. In the laboratory frame, the contact discontinuity and the two shocks move to the right.

For $\sigma=1.0$ (red), the solution shows similar profiles $\left(\leftarrow S C S_{\rightarrow}\right)$ as for $\sigma=0.1$. The FS is stronger (due to a higher jump in pressure) and slower (more deceleration relative to the frame of the contact discontinuity), while the RS is weaker but faster. These features are expected from analytical work

\footnotetext{
7 Here, $t$ is in units of $x / c$ with $c=1$.
}

(ZK05; Giannios et al. 2008), and agree with one-dimensional relativistic magnetohydrodynamic (MHD) simulations (Mimica et al. 2007, 2008).

When the magnetization of the flow exceeds $\sigma=2.7$, the shock profiles change drastically (the significance of this particular value of $\sigma$ is discussed below). For $\sigma=10.0$ (green) and 20.0 (blue), a prominent left-going rarefaction wave $(\leftarrow R)$ is observed, instead of a left-going shock (see also Romero et al. 2005; Mimica et al. 2007). When the rarefaction wave propagates into the jet flow, density and gas pressure decrease, and the flow velocity increases. The terminal Lorentz factor of the left (jet) state and the FS region reaches $\gamma \sim 14$ for $\sigma=10$ and $\gamma>16$ for $\sigma=20$. This magnetic acceleration mechanism stems from the magnetic pressure in the flow. ${ }^{8}$

This magnetic acceleration mechanism is solely an MHD effect and requires the magnetic field to generate a rarefaction

\footnotetext{
8 We note that Romero et al. (2005) and Mimica et al. (2007) also discovered the rarefaction wave regime discussed in this Letter, but did not investigate the magnetic acceleration mechanism and its astrophysical implications in detail.
} 

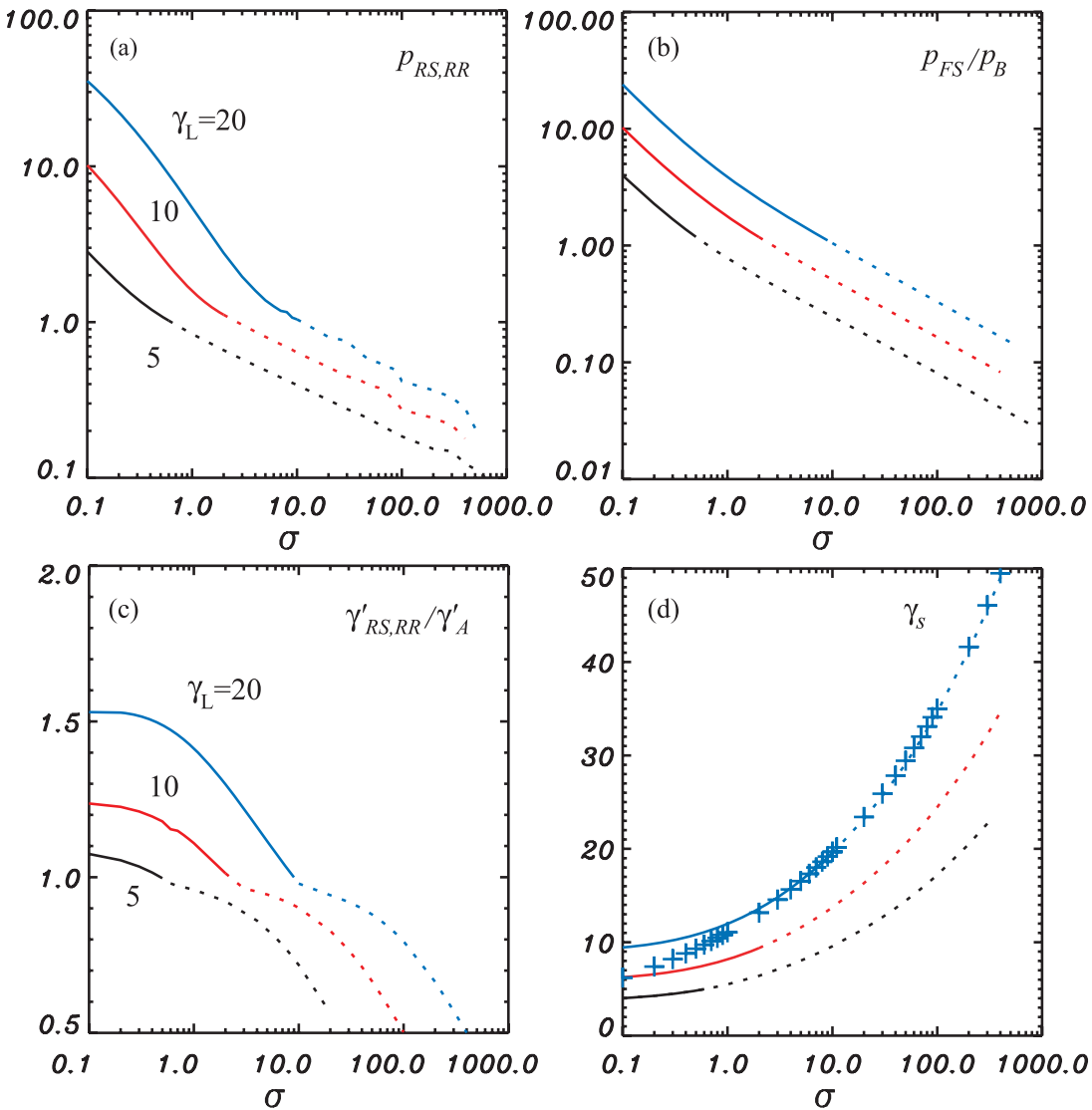

Figure 2. $\sigma$ dependences of (a) gas pressure in the region through which the reverse shock (RS; solid lines)/rarefaction wave (RR; dotted lines) has propagated; (b) the ratio of the gas pressure in the forward shock region to the magnetic pressure in the flow; (c) the ratio of the Lorentz factor of the propagating reverse shock/rarefaction wave to the Alfvén Lorentz factor in the rest frame of the fluid; (d) the maximum Lorentz factor in the shocked region, in the exact solution. Different initial Lorentz factors have been calculated: $\gamma_{L}=5$ (black), 10 (red), and 20 (blue). Crosses are the values of the estimated terminal Lorentz factor in the $\gamma_{L}=20$ case according to Equation (2). A constant flow density is adopted, so that the total initial energy density of the flow increases with $\sigma$.

wave. This is different from the HD/MHD boost mechanism proposed by Aloy \& Rezzolla (2006), and further investigated by Mizuno et al. (2008) and Aloy \& Mimica (2008). The HD/MHD mechanism is a purely relativistic mechanism, which invokes a relativistic flow perpendicular to the propagation direction of the rarefaction wave. The mechanism discussed here occurs even in the Newtonian case, and acts parallel to the propagation direction of the rarefaction wave. In general, the acceleration efficiency is smaller than that of the HD/MHD boost mechanism (see Section 3.3 for more discussions).

\subsection{Conditions for Reverse Shock or Magnetic Acceleration}

The magnetic pressure profiles (dotted lines) in Figure 1(b) reveal the physical condition required for the transition from a reverse shock to a rarefaction wave. It is evident that the magnetic pressure increases as $\sigma$ increases. In the reverse shock cases $(\sigma=0.1,1)$ the upstream magnetic pressure is lower than the gas pressure in the forward shock, while in the rarefaction wave cases $(\sigma=10,20)$ the upstream magnetic pressure exceeds the gas pressure in the FS. Thus, the balance between the upstream magnetic pressure in the unshocked flow region and the FS gas pressure in the shocked medium (ZK05; Romero et al. 2005) provides the condition separating the two regimes. This condition can be derived analytically (see also ZK05). For the interaction between a relativistic flow and an external medium, there exist four physically distinct regions: (1) unshocked medium, (2) shocked medium, (3) shocked flow, and (4) unshocked flow. Hereafter, $Q_{i}$ denotes the value of a quantity " $Q$ " in region " $i$ ". From the relativistic shock jump conditions with $\Gamma=4 / 3$, one can write $u_{2} / \rho_{2} c^{2}=\left(\gamma_{2}-1\right) \simeq \gamma_{2}$ and $\rho_{2} / \rho_{1}=4 \gamma_{2}+3 \simeq 4 \gamma_{2}$. A constant speed across the contact discontinuity requires $\gamma_{2}=\gamma_{3}$, and the relation between the gas pressure and the internal energy gives $p_{2}=u_{2} / 3$. Thus, the thermal pressure generated in the FS region is $p_{2}=(1 / 3)\left(\gamma_{2}-1\right)\left(4 \gamma_{2}+3\right) \rho_{1} c^{2} \simeq(4 / 3) \gamma_{2}^{2} \rho_{1} c^{2}$. The pressure balance condition is $B_{4}^{2} / 8 \pi \gamma_{4}^{2} \sim p_{2}$, because in pressure balance there is no reverse shock or rarefaction wave, so that regions 4 and 3 are matched as $B_{4} \simeq B_{3}$ and $\gamma_{4} \simeq \gamma_{3}=\gamma_{2}$. Using the definition $\sigma \equiv \sigma_{4}=B_{4}^{2} / 4 \pi \gamma_{4}^{2} \rho_{4} c^{2}$, one can derive a critical $\sigma_{c}$ value:

$$
\sigma_{c}=\frac{2}{3}\left(\gamma_{4}-1\right)\left(4 \gamma_{4}+3\right) \frac{\rho_{1}}{\rho_{4}} \simeq \frac{8}{3} \gamma_{4}^{2} \frac{\rho_{1}}{\rho_{4}}=\frac{8}{3} \gamma_{L}^{2} \frac{\rho_{R}}{\rho_{L}} .
$$

The condition for the existence of a reverse shock is $\sigma<\sigma_{c}$, which is Equation (31) of ZK05. The condition for a rarefaction wave and magnetic acceleration is $\sigma>\sigma_{c}$. We adopted $\rho_{1}=\rho_{R}=1.0, \rho_{4}=\rho_{L}=10^{2}$, and $\gamma_{4}=\gamma_{L}=10.0$, so that the critical value is $\sigma_{c} \simeq 2.7$. Our calculations indicate that $\sigma_{c}$ marks the transition point where neither a reverse shock nor a rarefaction wave is established (yellow lines in Figure 1). To verify this for a larger parameter space, we investigate the $\sigma$ dependences of various quantities in detail. Figure 2(a) shows the gas pressure in the region through which the reverse shock/ rarefaction wave has propagated. Initial Lorentz factors are $\gamma_{L}=5,10$, and 20, respectively. For all cases, we fix the flow density at $\rho_{L}=10^{2}$ and increase $B$ (hence $\sigma$ ). The total initial 

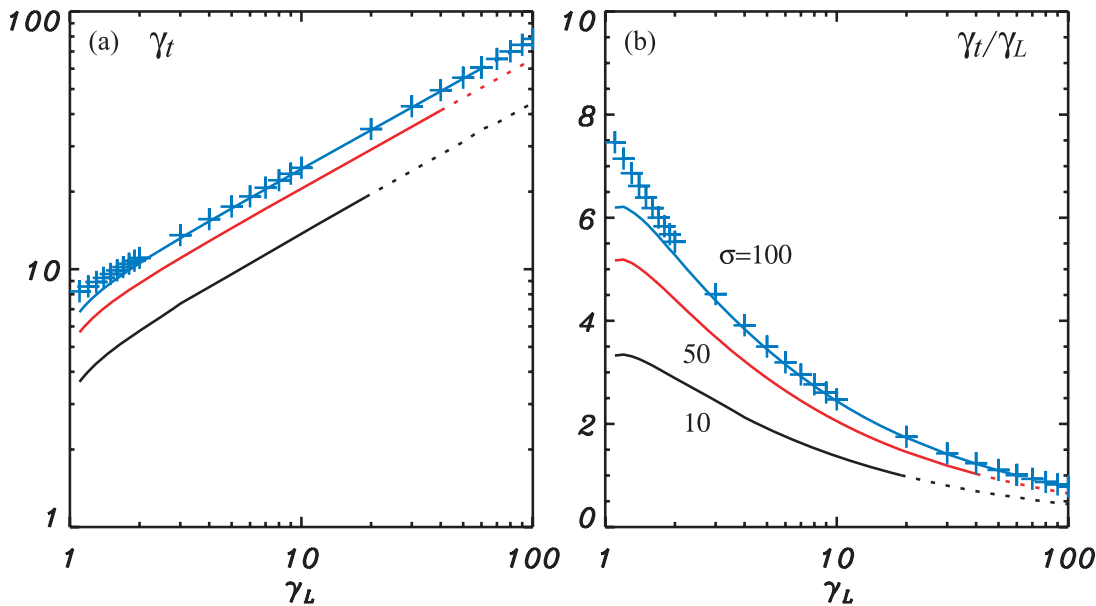

Figure 3. Dependences of (a) the terminal Lorentz factor $\gamma_{t}$ and (b) the acceleration efficiency $\gamma_{t} / \gamma_{L}$ on the initial Lorentz factor $\gamma_{L}$. Solid lines are for the RR regime and dotted lines are for the RS regime. Different initial magnetizations have been calculated: $\sigma=10$ (black), 50 (red), and 100 (blue). Crosses are values estimated with Equation (2) for $\sigma=100$.

energy density of the flow increases with $\sigma$. In all cases, the gas pressure decreases with $\sigma$ smoothly without a sharp transition from the RS regime (solid lines) to the reverse rarefaction wave regime (dotted lines). The critical magnetization parameters are $\sigma_{c} \simeq 0.7,2.7,10.6$ for $\gamma_{L}=5,10,20$, respectively, derived from the analytical solution of Equation (1). We note that in the RS regime, the strength of the shock decreases rapidly with increasing $\sigma$. The critical magnetization parameter $\sigma_{c}$ increases with $\gamma_{L}$, so that an RS can exist in the high- $\sigma$ regime if $\gamma_{L}$ is sufficiently large (see also ZK05).

Another commonly invoked RS condition states that the shock speed in the fluid frame (region 4) is higher than the speed of the Alfvén wave, i.e. $\gamma_{\mathrm{RS}, \mathrm{RR}}^{\prime}>\gamma_{A}^{\prime}$, where $\gamma_{\mathrm{RS}, \mathrm{RR}}^{\prime}=$ $\left(\gamma_{4} / \gamma_{\mathrm{RR}, \mathrm{RS}}+\gamma_{\mathrm{RR}, \mathrm{RS}} / \gamma_{4}\right) / 2, \gamma_{A}^{\prime}=(1+\sigma)^{1 / 2}$, and $\gamma_{\mathrm{RS}, \mathrm{RR}}$ is the Lorentz factor of the reverse shock or reverse rarefaction wave in the laboratory frame, which can be calculated using the exact solution of Giacomazzo \& Rezzolla (2006). Giannios et al. (2008) claim that this condition is different from the pressure balance condition. However, in Figures 2(b) and (c), we present the ratios of gas pressure in the FS region to magnetic pressure in the flow, $p_{\mathrm{FS}} / p_{B}$, and $\gamma_{\mathrm{RS}, \mathrm{RR}}^{\prime} / \gamma_{A}^{\prime}$, and find that both ratios reach unity at the same critical value $\sigma_{c}$. This suggests that the two reverse shock conditions have the same physical origin, at least for the one-dimensional model studied here.

\subsection{Terminal Lorentz Factor and Magnetic Acceleration Efficiency}

To better understand the magnetic acceleration mechanism, we plot the Lorentz factor as a function of $\sigma$ in Figure 2(d). For the magnetic acceleration case, this is the terminal Lorentz factor after acceleration. Because of the dependence of $\sigma_{c}$ on $\gamma_{L}$, a higher $\sigma$ is needed to achieve acceleration for a higher $\gamma_{L}$. The terminal Lorentz factor can be estimated analytically by requiring that the thermal pressure in the FS region balances the magnetic pressure in the region through which the rarefaction wave has propagated. For the terminal Lorentz factor $\gamma_{t}$, this condition can be expressed (roughly) as $B_{3}^{2} / 8 \pi \gamma_{t}^{2}=(1 / 3)\left(\gamma_{t}-1\right) \times$ $\left(4 \gamma_{t}+3\right) \rho_{1} c^{2} \simeq(4 / 3) \gamma_{t}^{2} \rho_{1} c^{2}$. From the definition of $\sigma$ with $B_{4} \simeq B_{3}$, this becomes

$$
\gamma_{t} \simeq\left(\frac{3 \gamma_{4}^{2} \sigma \rho_{4}}{8 \rho_{1}}\right)^{1 / 4}
$$

Crosses in Figure 2(d) denote values of terminal Lorentz factors calculated from Equation (2) for model parameters, $\gamma_{4}=\gamma_{L}=20, \rho_{1}=\rho_{R}=1.0$, and $\rho_{4}=\rho_{L}=10^{2}$, in good agreement with the exact solution of the Riemann problem in the reverse rarefaction wave regime.

To investigate the acceleration efficiency, we present in Figure 3 the terminal Lorentz factor $\gamma_{t}$, and its ratio to the initial Lorentz factor $\left(\gamma_{t} / \gamma_{L}\right)$ as a function of the initial flow Lorentz factor $\gamma_{L}$. While a flow with a higher initial Lorentz factor reaches a higher terminal Lorentz factor, a lower initial Lorentz factor implies a higher acceleration efficiency. From Equation (2) it follows that $\gamma_{t} / \gamma_{4} \simeq\left(3 \sigma \rho_{4} / 8 \rho_{1}\right)^{1 / 4} \gamma_{4}^{-1 / 2}$, in good agreement with the exact solution of the Riemann problem in the relativistic regime.

\section{SUMMARY AND DISCUSSION}

We solved the one-dimensional Riemann problem for the deceleration of an arbitrarily magnetized relativistic flow in a static, unmagnetized medium. For the same initial Lorentz factor, the reverse shock becomes progressively weaker with increasing $\sigma$, turning into a rarefaction wave when $\sigma \geqslant \sigma_{c}$, at which point the magnetic pressure in the flow is balanced by the thermal pressure in the forward shock. In the rarefaction wave regime, material in the FS region is accelerated due to the strong magnetic pressure in the flow. This magnetic acceleration mechanism may thus play an important role in the dynamics of strongly magnetized, relativistic flows.

Numerical MHD simulations (e.g., Koide et al. 1999, 2000; Nishikawa et al. 2005; Hardee et al. 2007) are essential to understand magnetized relativistic jets. We performed one-dimensional special relativistic MHD simulations of a relativistic flow propagating in an external medium using the RAISHIN code (Mizuno et al. 2006). The simulation results are in good agreement with the exact solution (Giacomazzo \& Rezzolla 2006), serving as a test of the RAISHIN code. As MHD simulations can tackle problems for which an exact solution is not known, we plan to utilize RAISHIN to solve more realistic configurations (e.g., relativistic shells with a finite width and conical geometry, as often envisaged in the GRB problem).

The magnetic acceleration mechanism discussed here also applies in the Newtonian MHD limit. The transition point from a reverse shock to a rarefaction wave is then also given by 
the pressure balance condition, and the terminal velocity of the flow can be estimated from the Newtonian shock jump condition as $v_{t}=c_{s 1}\left(p_{2} / p_{1}-1\right) \sqrt{2 / \Gamma /\left[(\Gamma+1)\left(p_{2} / p_{1}\right)+(\Gamma-1)\right]}$, where $c_{s 1}=\left(\Gamma p_{1} / \rho_{1}\right)^{1 / 2}$ is the sound speed in the upstream medium (see also Hawley et al. 1984). Defining $\sigma=$ $\left(B_{4}^{2} / 8 \pi\right) /\left(\rho_{4} v_{4}^{2} / 2\right)$ in the Newtonian limit, one can derive the terminal velocity of the flow, $v_{t}$, determined by balance between the magnetic pressure and the pressure in the forward shock region, $p_{2}=B_{3}^{2} / 8 \pi \simeq B_{4}^{2} / 8 \pi=\sigma \rho_{4} v_{4}^{2} / 2$. For a strong shock $\left(p_{2} \gg p_{1}\right)$, the terminal velocity can be approximated as $v_{t} \simeq \sqrt{\sigma\left(\rho_{4} / \rho_{1}\right) /(\Gamma+1)} v_{4}$. For $v_{t}=v_{4}$, one derives $\sigma_{c} \simeq(\Gamma+1) \rho_{1} / \rho_{4}$. For $\Gamma=5 / 3$, typical for nonrelativistic shocks, this expression is consistent with Equation (1) for $\gamma_{L}=1$. Although the general physics is the same, the dependence of the terminal velocity $\left(v_{t} / c\right) \gamma_{t}$ is different in the Newtonian and relativistic case.

Our results have implications for understanding the deceleration of strongly magnetized outflows, possibly present in GRBs and AGNs. Exact solutions indicate that the condition for the existence of a reverse shock is $\sigma<\sigma_{c}$, as suggested by ZK05 (see Giannios et al. 2008). The paucity of bright optical flashes in GRBs (e.g., Roming et al. 2006) may, among other interpretations, be attributed to highly magnetized flows. Furthermore, the magnetic acceleration mechanism discussed here suggests that $\sigma$ and $\gamma$ are not independent parameters at the deceleration radius. For high- $\sigma$ flows, the ejecta would experience magnetic acceleration at small radii, before reaching the coasting regime, so that the coasting Lorentz factor (i.e., the "initial" Lorentz factor for the afterglow) is at least the "terminal" Lorentz factor defined by Equation (2). As a result, the high- $\sigma$ and low- $\gamma$ part of parameter space is suppressed. This implies that some region in the $\xi-\sigma$ parameter space ${ }^{9}$ of GRB models (ZK05; Giannios et al. 2008) is suppressed as well. Here we only focus on one-dimensional models with Cartesian geometry. Implications for GRB models will be discussed in more detail when this Riemann problem is solved in the conical jet geometry.

Variable emission (down to minute timescales) observed in some TeV blazars suggests very high Lorentz factors in AGN jets (Aharonian et al. 2007). Models for the production of $\mathrm{TeV}$ emission appeal to high Lorentz factor jet cores surrounded by lower Lorentz factor sheaths (e.g., Ghisellini et al. 2005) or rapid jet deceleration (Georganopoulos et al. 2005) in order to reconcile the required jet Lorentz factors with lower Lorentz factors suggested by proper motion studies. Our results suggest the possibility of magnetic acceleration occurring where highly magnetized jet material overtakes more weakly magnetized jet material. In this case the magnetically accelerated Lorentz factor behind the forward shock can significantly exceed the Lorentz factor of the overtaken jet material.

In this study we considered a static, unmagnetized medium but for a weakly magnetized medium our conclusions hold as well. However, the conclusions will not apply in the very large $\sigma$ regime when the underlying MHD approximation breaks down, e.g., $\sigma \sim$ several 100 s in the problem of GRBs (Spruit et al. 2001; Zhang \& Mészáros 2002).
We thank S. Kobayashi, H. Sol, and L. Rezzolla for helpful comments. Y.M. and B.Z. acknowledge NASA NNG05GB67G, NNG05GB68G, and NNX08AE57A for partial support during Y.M.'s stay at UNLV. Y.M., P.H., and K.I.N. acknowledge partial support by NSF AST-0506719, AST-0506666, NASA NNG05GK73G, NNX07AJ88G, and NNX08AG83G. B.G. thanks CSPAR-UAH/NSSTC for hospitality during the preparation of part of this work and acknowledges the DFG grant SFB/Transregio 7 for partial support. S.N. is partially supported by Grants-in Aid for Scientific Research of the Japanese Ministry of Education, Culture, Sports, Science and Technology 19047004, 19104006, and 19740139. The simulations were performed on Columbia Supercomputer at NAS Division at NASA Ames Research Center and Altix3700 BX2 at YITP in Kyoto University.

\section{REFERENCES}

Aharonian, et al. (HESS collaborations) 2007, ApJ, 664, L71

Aloy, M. A., \& Mimica, P. 2008, ApJ, 681, 84

Aloy, M. A., \& Rezzolla, L. 2006, ApJ, 640, L115

Blandford, R. D. 1976, MNRAS, 176, 465

Blandford, R. D., \& Payne, D. G. 1982, MNRAS, 199, 883

Blandford, R. D., \& Znajek, R. L. 1977, MNRAS, 179, 433

Fan, Y.-Z., Dai, Z.-G., Huang, Y.-F., \& Lu, T. 2002, Chin. J. Astron. Astrophys., 2, 449

Fan, Y. Z., Wei, D. M., \& Wang, C. F. 2004, A\&A, 424, 477

Georganopoulos, M., Perlman, E. S., \& Kazanas, D. 2005, ApJ, 634, L33

Ghisellini, G., Tavecchio, F., \& Chiaberge, M. 2005, A\&A, 432, 401

Giacomazzo, B., \& Rezzolla, L. 2006, J. Fluid Mech., 562, 223

Giannios, D., Mimica, P., \& Aloy, M. A. 2008, A\&A, 478, 747

Gomboc, A., et al. 2008, ApJ, 687, 443

Hardee, P., Mizuno, Y., \& Nishikawa, K.-I. 2007, Ap\&SS, 311, 281

Hawley, J. F., Smarr, L. L., \& Wilson, J. R. 1984, ApJ, 277, 296

Kennel, C. F., \& Coroniti, F. V. 1984, ApJ, 283, 694

Kobayashi, S., Piran, T., \& Sari, R. 1999, ApJ, 513, 669

Koide, S., Meier, D. L., Shibata, K., \& Kudoh, T. 2000, ApJ, 536, 668

Koide, S., Shibata, K., \& Kudoh, T. 1999, ApJ, 522, 727

Kumar, P., \& Panaitescu, A. 2003, MNRAS, 346, 905

Lovelace, R. V. E. 1976, Nature, 262, 649

Lyutikov, M. 2006, New J. Phys., 8, 119

Lyutikov, M., \& Blandford, R. D. 2003, arXiv:astro-ph/0312347

Mészáros, P., \& Rees, M. J. 1997, ApJ, 482, L29

Mimica, P., Aloy, M. A., \& Müller, E. 2007, A\&A, 466, 93

Mimica, P., Giannios, D., \& Aloy, M. A. 2008, A\&A, in press (arXiv:0810.2961)

Mizuno, Y., Hardee, P., Hartmann, D. H., Nishikawa, K.-I., \& Zhang, B. 2008, ApJ, 672, 72

Mizuno, Y., Nishikawa, K.-I., Koide, S., Hardee, P., \& Fishman, G. J. 2006, in Proc. VI Microquasar Workshop: Microquasars and Beyond, ed. Tomaso (Trieste: SISSA), 45

Nishikawa, K.-I., Richardson, G., Koide, S., Shibata, K., Kudoh, T., Hardee, P., \& Fishman, G. J. 2005, ApJ, 625, 60

Romero, R., Martí, J. M., Pons, J. A., Ibáñez, J. M., \& Miralles, J. A. 2005, J. Fluid Mech., 544, 323

Roming, P. W. A., et al. 2006, ApJ, 652, 1416

Sari, R., \& Piran, T. 1995, ApJ, 455, L143

Spruit, H. C., Daigne, F., \& Drenkhahn, G. 2001, A\&A, 369, 694

Thompson, C. 1994, MNRAS, 272, 480

Usov, V. V. 1992, Nature, 357, 472

Vlahakis, N., \& Königl, A. 2003, ApJ, 596, 1080

Vlahakis, N., \& Königl, A. 2004, ApJ, 605, 656

Zhang, B., \& Kobayashi, S. 2005, ApJ, 628, 315 (ZK05)

Zhang, B., Kobayashi, S., \& Mészáros, P. 2003, ApJ, 595, 950

Zhang, B., \& Mészáros, P. 2002, ApJ, 581, 1236

\footnotetext{
$9 \xi$ is defined as $\sqrt{r_{\mathrm{dec}} / r_{s}}$ (Sari \& Piran 1995), where $r_{\mathrm{dec}}$ is the deceleration radius where the ejecta accumulate from the external medium a mass $\gamma_{0}^{-1}$ times their own mass, and $r_{s}$ is the spreading radius where the width of the ejected shell starts to increase due to propagation of a sound wave.
} 